\title{
UAV-BASED PHOTOGRAMMETRIC POINT CLOUDS - TREE STEM MAPPING IN OPEN STANDS IN COMPARISON TO TERRESTRIAL LASER SCANNER POINT CLOUDS
}

\author{
A. Fritz ${ }^{\mathrm{a}}$, T. Kattenborn ${ }^{\mathrm{a}}$, B. Koch ${ }^{\mathrm{a}}$ \\ ${ }^{a}$ Albert-Ludwigs-University Freiburg, Chair for remote sensing and LIS, 79106 Freiburg , Germany \\ (andreas.fritz, teja.kattenborn, barbara.koch) \\ @ felis.uni-freiburg.de
}

KEY WORDS: UAV, point clouds, CMVS/PMVS, structure from motion, single tree detection, forest inventory

\begin{abstract}
:
In both ecology and forestry, there is a high demand for structural information of forest stands. Forest structures, due to their heterogeneity and density, are often difficult to assess. Hence, a variety of technologies are being applied to account for this "difficult to come by" information. Common techniques are aerial images or ground- and airborne-Lidar. In the present study we evaluate the potential use of unmanned aerial vehicles (UAVs) as a platform for tree stem detection in open stands. A flight campaign over a test site near Freiburg, Germany covering a target area of $120 \times 75\left[\mathrm{~m}^{2}\right]$ was conducted. The dominant tree species of the site is oak (quercus robur) with almost no understory growth. Over 1000 images with a tilt angle of $45^{\circ}$ were shot. The flight pattern applied consisted of two antipodal staggered flight routes at a height of $55[\mathrm{~m}]$ above the ground. We used a Panasonic G3 consumer camera equipped with a $14-42[\mathrm{~mm}]$ standard lens and a 16.6 megapixel sensor. The data collection took place in leaf-off state in April 2013. The area was prepared with artificial ground control points for transformation of the structure-from-motion (SFM) point cloud into real world coordinates. After processing, the results were compared with a terrestrial laser scanner (TLS) point cloud of the same area. In the $0.9[\mathrm{ha}]$ test area, 102 individual trees above 7[cm] diameter at breast height were located on in the TLS-cloud. We chose the software CMVS/PMVS-2 since its algorithms are developed with focus on dense reconstruction. The processing chain for the UAV-acquired images consists of six steps: a. cleaning the data: removing of blurry, under- or over exposed and off-site images; b. applying the SIFT operator [Lowe, 2004]; c. image matching; d. bundle adjustment; e. clustering; and f. dense reconstruction. In total, 73 stems were considered as reconstructed and located within one meter of the reference trees. In general stems were far less accurate and complete as in the TLS-point cloud. Only few stems were considered to be fully reconstructed. From the comparison of reconstruction achievement with respect to height above ground, we can state that reconstruction accuracy decreased in the crown layer of the stand. In addition we were cutting $50[\mathrm{~cm}]$ slices in z-direction and applied a robust cylinder fit to the stem slices. Radii of the TLS-cloud and the SFM-cloud surprisingly correlated well with a Pearson's correlation coefficient of $r=0.696$. This first study showed promising results for UAV-based forest structure modelling. Yet, there is a demand for additional research with regard to vegetation stages, flight pattern, processing setup and the utilisation of spectral information.
\end{abstract}

\section{INTRODUCTION}

\subsection{Utilization of UAVs in the environmental sector}

Recently, unmanned aerial vehicles (UAVs) have evolved to offthe-shelf platforms for remote sensing applications and photogrammetric data acquisition. Early work started back in the 2000 with mainly model air-planes and helicopters [Hongoh et al., 2001, Hunt et al., 2005]. UAV based digital surface models and ortho images have already been successfully evaluated and proved to be sound products. Moreover UAVs facilitate data acquisition with a high spatial and temporal resolution, also referred to as hyperspatial and hypertemporal, [Lucieer et al., 2012], which yielded a lot of attention regarding the applicability of UAVs in the environmental sector [Haala et al., 2011]. Compared to other platforms such as airborne Lidar, relative low costs for acquisition of information and high flexibility with such devices led to intense research in that field. UAVs of different size and technology have been used in applications such as precision agriculture [Valente et al., 2013, Zhang and Kovacs, 2012], fire monitoring [Merino et al., 2012] and forest characterization [Tao et al., 2011, Wallace et al., 2012]. Payload used range from off-the-shelf digital cameras [Rosnell and Honkavaara, 2012] to FLIR systems [Turner et al., 2011] and laser scanners [Wallace et al., 2012]. In addition more and more efficient algorithms for transforming the enormous amount of collected images into 3D information become available for public through web services or open source tools. Examples for the first are Microsoft Photosynth or 123D
Catch Beta for free processing. Bundler [Snavely et al., 2006] and CMVS/PMVS-2 [Furukawa and Ponce, 2010a] are options for the latter. Output of the programs is a sparse to dense point cloud generated through image matching. The point cloud carries additional information such as color (taken from the images) or point-normals (estimated during the processing).

\subsection{Dense 3D reconstruction for forest stand parameters}

In both ecology and silviculture, there is a high demand for accurate forest structure information. Modern forest resource planning, applications such as industrial wood flow optimization, require accurate inventories of forest stands, including information as diameter at breast height (DBH) distribution or tree density [Moberg and Nordmark, 2006] and tree quality parameters. Likewise high resolution 3D-information is needed in many scientific fields and essential for accurate mapping of vegetation biomass [Frolking et al., 2009], habitat quality [Vierling et al., 2008] or carbon storage [Asner, 2009]. To obtain this three-dimensional information Lidar is widely and commercially used in forest inventories [Hyyppä et al., 2008]. However, aerial laser scanning (ALS) exhibits high area coverage, but relatively high costs and lower point densities, whereas the latter is limiting tree detection accuracy [Tesfamichael et al., 2009]. In contrast to ALS, terrestrial laser scanning (TLS) produces very high point densities, although decreasing with increasing distance to the sensor and covering a relatively small areas. Point cloud generation based on UAV platforms and image matching could possibly fill in the gap between ALS and TLS, accessing/covering quite large areas, 
delivering high point densities for accurate detections, while requiring relatively little resources.

Attempts to mount Lidar sensors on UAVs have shown great potential. However in [Wallace et al., 2012] several limitations are indicated, e.g. the generation of accurate and dense point clouds is only possible at quite low altitudes (below $50 \mathrm{~m}$ ). This restricts applications in stands with taller trees. Area coverage is furthermore limited as the relatively high weight of suitable Lidar sensors only allows for shorter flight durations (employing a conventional octocopter). [Eisenbeiss, 2009] use a large size UAV (Aeroscout Scout B1-100) with a maximum take-off weight of $75[\mathrm{~kg}]$ equipped with a airborne laser scanner Riegle LMS-Q160 for initial testing. Such settings are likely to produce results comparable to ALS campaigns, but with higher point densities. Nevertheless, costs are considerably higher.

A technique to extract 3D-information of UAV flights is given by the structure-from-motion (SFM) processing chain, which produces point clouds on the basis of feature matches within overlapping images. Several studies depicted a high potential of the combination of UAV image flights and the SFM processing chain, particularly with regard to urban objects and terrain modeling [Remondino et al., 2011]. By now fewer attempts have been conducted to extract vegetation structure or specifically information on forest stands. Previous studies indicated difficulties and limitations of this technique [Harwin and Lucieer, 2012, Rosnell and Honkavaara, 2012] in complex and heterogeneous spatial structures such as vegetation. Thus, 3D-reconstruction of vegetation requires an approach which differs from the above mentioned objects in terms of the data acquisition setup and data processing and its parameters. In this initial study, we present and evaluate an approach to reconstruct and automatically detect individual trees, based on an aerial survey and processing setup which is adjusted to broad leaf forest stands.

\section{MATERIAL AND METHODS}

\subsection{Study Site}

The study site is located in an old oak (Quercus robur 90\%) dominated stand close to Freiburg I. Brsg. Germany (48 $0^{\prime} 51$ $\left.7^{\circ} 44^{\prime} 39\right)$. The stand has little under growth and a low stem density. Relevant species beside oak are hornbeam (Carpinus betulus 10\%) and maple (Acer pseudoplatanus). The entire area $(36.4[h a])$ is protected due its near to nature forest community with a significant portion of standing dead wood, bird's nests and bat appearance. In total the study site covers only $0.9[\mathrm{ha}]$ $\left(75 \times 125 \mathrm{~m}^{2}\right)$. Figure 1 shows an overview of the site.

\subsection{TLS survey of the study site}

To compare the results of the UAV-data eight terrestrial laser scans (Faro Focus 3D 120) were carried out during leaf-off state (date of scan campaign 12.02.2013). Five spheres for registration of the scans were placed in the target area. Mean registration error of the eight scans was $0.0035[\mathrm{~m}]$. After registration the point density was very high (125Mio points) due to massive overlap of the different scans. To speed up processing time two filters were applied: a) a voxelgrid filter with a grid size of $1[\mathrm{~cm}]$ and b) an outlier removal to eliminate ghost and isolated points. Furthermore, the entire point set was cropped to the study area. In the end 22Mio points have been used for further processing.

\subsection{UAV setup and data acquisition}

With respect to the study area and the processing chain the following requirements of the UAV-platform were identified: a) capability of starting and landing within the forest through the canopy

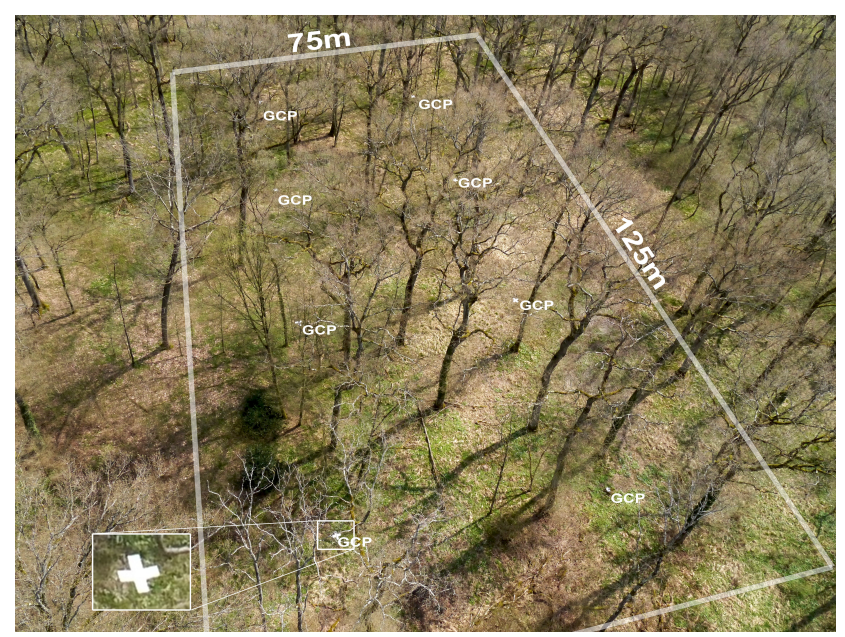

Figure 1: View of the study area including ground control points (highlighted in the lower left).

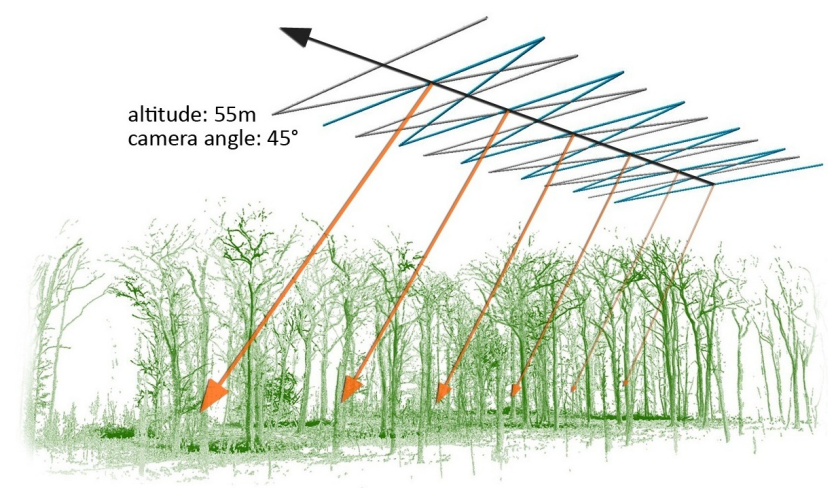

Figure 2: The blue and grey zigzag-lines show the flight path of the UAV. Orange arrows indicate the direction of the camera and the camera angle of $45^{\circ}$.

b) flying at low altitudes close to the canopy top and c) ability of performing small meshed flight patterns. In accordance with these criteria an octocopter was chosen as the UAV-platform, which is based on a modified MK Okto2 (Highsystems GmbH). A standard consumer camera (Panasonic Lumix G3) was used as sensor. It was fixed on a flexible mount which enables tilting the camera vertically and horizontally. This also compensates changes of orientation while cruising. The camera was triggered at a pulse rate of $1.4[\mathrm{~Hz}]$. In general a UAV-based Lidar would be a potential sensor. However, the main constraint for our study was seen in the current price for such device.

After evaluation of the different flight patterns and the corresponding 3D reconstructions as well as camera settings a double zigzag pattern was chosen (see Figure 2). It consists of two flights shifted along the direction of view.

Two final image flights were performed on a slightly cloudy afternoon in mid April 2013. Image acquisition took place at a height above ground of $55[\mathrm{~m}]$ and a cruising speed of $2.5[\mathrm{~m} / \mathrm{s}]$. The horizontal viewing direction was kept stable to $118^{\circ}$. In total 1129 images were taken. Even with the zigzag-pattern, the vertical camera angle of $45^{\circ}$ was held constant. The focal length of $14[\mathrm{~mm}]$, an aperture of $F=9$, ISO film speed equivalent of 800 and a shutter speed of $1 / 800[\mathrm{sec}]$ were set on the camera.

\subsection{UAV-data processing}

Pictures taken by the UAV were manually inspected regarding blur, under- or over-exposure, visible horizon and off-site. Even- 


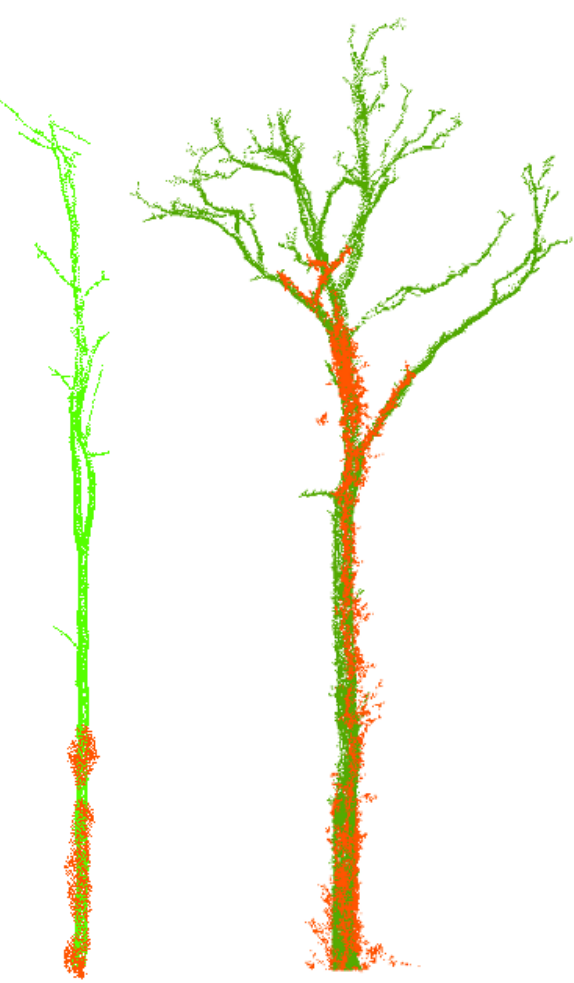

Figure 3: TLS-trees (green) and reconstructed trees (red). Left shows a partly reconstructed smaller tree. Right shows a tree with a fully reconstructed stem and parts of the crown.

though, the used algorithms are known to be robust to changing image quality [Lowe, 2004], orthophoto generation is affected by the image choice. The cleaned set of images is processed with a typical structure from motion tool chain. In our case with VisualSFM developed by [Furukawa and Ponce, 2010b]. It pipes the results of the different processing steps to the succeeding tool and visualizes intermediate results. In five steps, a dense point cloud is generated:

1. applying the SIFT operator (Lowe, 2004): the semi invariant feature transform detector is used to automatically generate tie points for each image;

2. image matching: with the help of detected SIFT-features each image is matched with its corresponding images;

3. bundle adjustment: a multicore bundle adjustment [Wu et al., 2011] is applied to estimate camera parameters;

4. clustering: clustering the image set for efficient dense reconstruction and;

5. dense reconstruction: generating point clouds from images and camera parameters.

Each step can be manipulated by a variety of parameters. Choosing suitable settings for each step is a complicated procedure, requiring a high number of tests. In addition, the settings influence each other and therefore a systematic analyses of the effect of a single parameter is difficult. We have chosen a trade of between reconstruction completeness and correctness. Settings towards a high correctness in terms of a high spatial accuracy of the reconstructed 3D-points are made at the expenses of a preferably comprehensive reconstruction. In Table 1 basic characteristics of structure from motion procedure are summarized.

\begin{tabular}{|l|r|}
\hline Characteristic & Value \\
\hline Total number of images taken & 1129 \\
Number of images used for reconstruction & 862 \\
Min. images for 3D-reconstruction & 5 \\
3D-Points after dense reconstruction & $33.5 \mathrm{Mio}$ \\
3D-Points reduction to target area & $15.7 \mathrm{Mio}$ \\
\hline
\end{tabular}

Table 1: Summary of the structure from motion reconstruction.

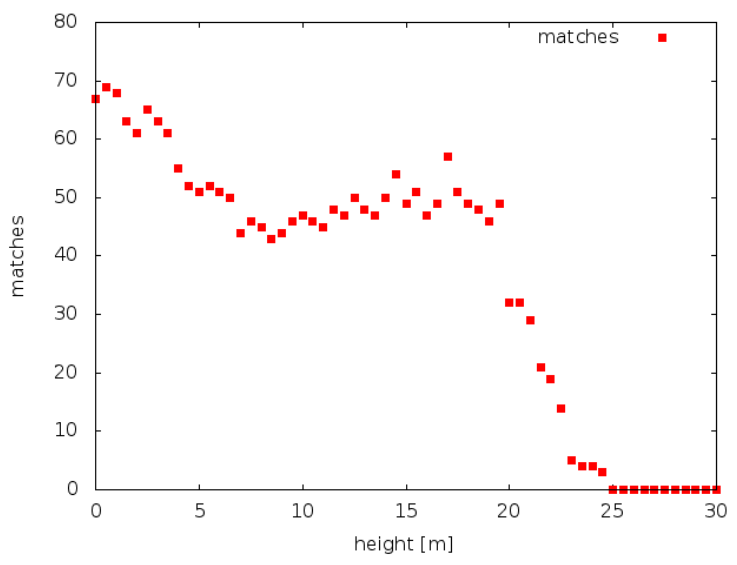

Figure 4: Matches between reference and reconstructed trees in relation to height above ground.

\subsection{Data Analysis}

Data analysis was conducted in five steps: 1 . registration of the TLS and UAV-point cloud; 2. horizontal slicing of both clouds; 3. extraction of coherent point clusters; 4 . stem radius estimation; 5 . appearance check of UAV-based clusters in TLS-based clusters.

registration \& cleaning Ground control points were used to estimate transformations parameters of Helmert-transformation. With seven ground control points a RMSE of $0.12[\mathrm{~m}]$ could be achieved. With the transformation, the UAV-Point cloud got scaled by the factor 2.43. To eliminate outliers and single points we filtered the point cloud with a neighbour relation approach described in [Rusu et al., 2008]. Figure 5 shows a view into the cleaned point cloud.

horizontal slicing The test site consist of relatively flat terrain which allows a simple $\mathrm{z}$-value threshold for horizontal slicing as height layer creation. Identical slices of $50[\mathrm{~cm}]$ thickness were cut out of both point clouds.

extraction of coherent point clusters The slices contain a high amount of spurious points. However, tree parts are characterized by dense accumulation of points. We extracted coherent point clusters by defining cluster criteria regarding minimum and maximum points per cluster as well as maximal distance between cluster points. It turned out that best results could be achieved in a range of $40 \ldots 10.000$ points per cluster.

radius estimation Each cluster of $50[\mathrm{~cm}]$ thickness was used as input point set for a RANSAC based cylinder fit. To ensure that the model cylinder fits the point set we defined criteria for accepting estimated parameters: a) radius must be between $0.05 \ldots 1.0[\mathrm{~m}]$; b) the axis of the cylinder must be in direction of $\mathrm{z}$ with a maximum deviation of $30^{\circ}$; c) if after 5000 iterations no appropriate model coefficients can be found stop the iterating. To eliminate outliers, radii must be within $r_{\text {accept }} \leq \bar{r}+2 \sigma_{r}$. 


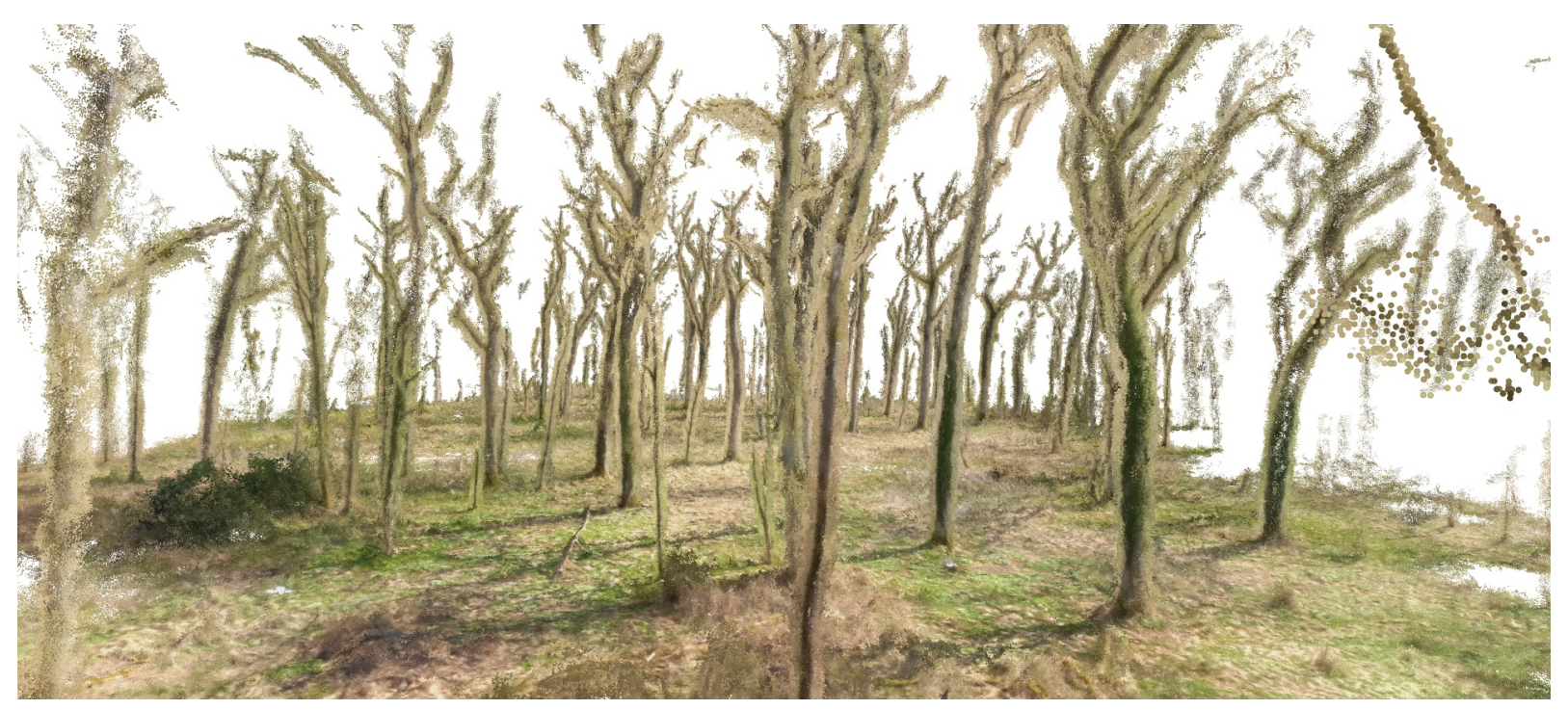

Figure 5: View into the cleaned SFM-point cloud.

appearance check As last step, we checked if the UAV-based stem cluster appeared in the TLS-based stem cluster by setting a maximal allowed distance of $1[\mathrm{~m}]$. If clusters from both point clouds were within $1[m]$ of each other we considered this segment as successfully reconstructed. In cases of $n: m$ pairs we achieve a $1: 1$ relationship while taking into account the estimated radius rather than the distance. For example for one TLS-segment, three SFM-segments were found within one meter, the segment with the radius closest to the TLS-segment's radius would be considered as a match.

\section{RESULTS}

Results are presented for tree detection and radius estimation separately, even though there is a relation between them. If a tree (segment) has been reconstructed, the radius estimation has been executed.

\subsection{Tree detection}

The test site contains 102 trees above $7[\mathrm{~cm}]$ diameter at breast height (dbh). Out of these $73(71 \%)$ were considered as reconstructed and located within one meter of the reference trees. This does not account for the different reconstruction output along the $\mathrm{z}$-axis, which varies thoroughly. A tree counts as detected if it has been reconstructed with at least 5000 points. The left tree in Figure 3 as example counts as detected. With respect to incomplete trees in the SFM-point cloud, the detection rate in dependent on the height layer was evaluated. Figure 4 illustrates the decrease of matches with increasing height and shows that at ca. $20[\mathrm{~m}]$ height a clear drop in matches occurs. This can be explained by the beginning of the crown, and therefore components (smaller branches), which are more difficult to reconstruct.

\subsection{Radius estimation}

With diameter estimation, a consistent trend could be observed. Figure 3 shows exemplary matched tree pairs. Figures 6 and 7 are based on the right tree. Radius estimation of the $50[\mathrm{~cm}]$ slices results in variations along the $\mathrm{z}$ axis. Similar to the matches, in lower parts of the stand a slightly better estimation is given. However, reliability decreases as the height location of increases.

Over all matches of slices of comparison of estimated radii of both data sets were made. The radii of the reconstructed slices seem to have a trend to be underestimated. The result of Pearson's test of linear correlation was significant at a two-tailed $\mathrm{p}$ value of $p<0.001$ and a correlation coefficient of $r=0.696$.

\section{DISCUSSION AND OUTLOOK}

Old open broad-leaf stands are in many ways attractive to foresters and environmentalists. On one hand they often represent high economic value (e.g. veneer production), and on the other such stands have a high ecological value as habitat for endangered species. Characterisation of such areas are highly favourable. UAVs can be seen as contributing towards new flexible and cheaper sensor platforms delivering high quality airborne spectral and 3Dinformation. We showed that in sparse forests single tree detection based on 3D-point clouds generated through image matching is possible. In the following sections we will discuss the results and the different settings made while conducting this study.

\subsection{Flight planning}

In several pre-studies, we experimented with different flight patterns and camera settings. Within traditional airborne image flights, pictures are taken in nadir direction towards ground and in parallel flight strips with usually $60 \%$ to $80 \%$ overlap. For the purpose of $3 \mathrm{D}$ - reconstruction of vertical structures, in this case single tree stems, nadir pictures are insufficient, trees are often occluded by the branches of taller trees and vertical surfaces.The ZigZagpattern with direction of view in $45^{\circ}$ performed very well regarding connectivity of the images and pixel coverage of the trees. Furthermore this pattern reduces shadow effects, where parts of the crown hide in worst-case an entire tree. However, one draw back in our setting is the fact that the direction of view is fixed into one direction (west to east). With this setting, we achieve a relatively complete coverage of the stand, but we are missing the back side of the scene. Thus, tree trunks cannot be reconstructed completely, they appear open on the back site. This effect can be observed in single TLS-scans in stands. Several studies show that a half reconstructed tree can still make reliable diameter estimations possible [Bienert et al., 2007, Liang et al., 2008, Lovell et al., 2011].

\subsection{Structure from motion process}

The open source software chain used in this study was able to process the image set and returned very promising results. Considering the vast amount of configurable parameters in each step, 


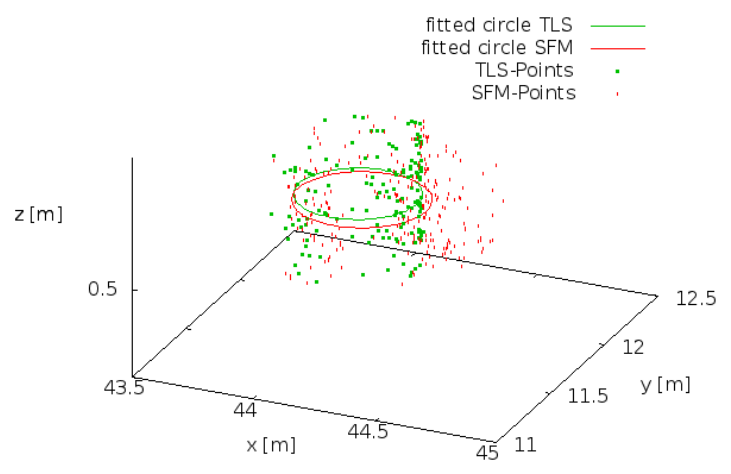
structed stem (red) and their corresponding fitted diameters.

evaluating the total performance of the system becomes difficult. Beginning with the first step - SIFT-detection - there has been evidence of a strong influence regarding specific settings [Lingua et al., 2009]. We conclude that standard settings of pmvs 2 are not optimal for reconstruction of strong jumps in distance to the camera. This especially applies to trees where small branches of the crowns are difficult to reconstruct due to their great drop in distance to the ground. It seems that increasing the pixels sampled (pmvs2: wsize) for calculating the photometric consistency score lead to better results. On the same hand lowering the threshold for accepting a reconstruction leads to more 3D-points as well. Finding the best combination is a cumbersome process and needs to further optimized.

\subsection{Reconstruction results}

In general, the reconstruction of the ground with mainly grass vegetation is, except in shadowed areas complete and seems to be accurate. However, a detailed evaluation was not part of this study. Tree reconstruction can be seen as partly successful. The variation of reconstruction along the height axis is influenced by the density of structures in the crown. This means that thinner branches will likely remain undetected than the up to 50 times wider tree trunks near to the ground. As discussed in [Rosnell and Honkavaara, 2012], we can confirm that the strong threedimensionality of forests poses challenges for SFM processing and data acquisition. After testing various flight patterns we can conclude that generally reconstruction becomes more challenging at lower altitudes, while at higher altitudes accuracy decreases due reduced ground sampling distance. Another issue identified is the fact that trees are rarely reconstructed in a connected point set, rather they include gaps in the trunk and crown. Reducing shadow-effects in the image set used for the SFM-process can minimise the problem at the cost of increasing complexity of flight planning.

\subsection{Radius estimation}

Results of the radius estimation were unexpectedly good. Robust, RANSAC based cylinder fitting proved to be able to cope fairly well with the high variation within the stem points. The tendency of overestimating radii can be explained with the relaxed threshold of accepting a reconstruction which leads to less accurate results.

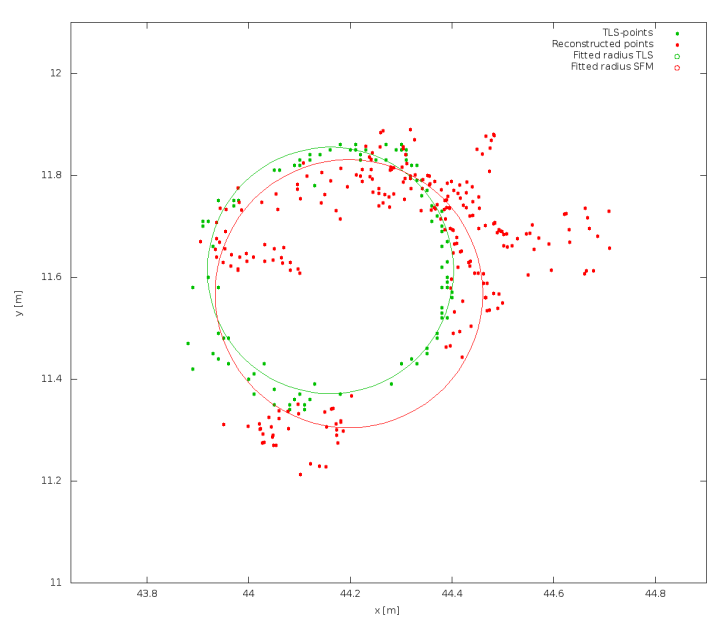

Figure 7: A 50[cm] slice of a TLS stem (green) and a reconstructed stem (red) and their corresponding fitted diameters projected on a plane.

\subsection{Future work}

This first study has shown good results and promising potential for UAV-based 3D-reconstruction of forest stands, yet more research has to be conducted with regard to the following topics:

flight pattern: Covering the scene with a coherent set of images, ensuring that the objects of interest are mapped from every side while minimizing shadow effects is very challenging. Testing new flight patterns can help to make up the forest. First attempts for agricultural sites can be found in [Valente et al., 2013].

processing setup: Many parameters in each processing step of the point cloud generation influence the results. Identification of the relevant parameters for forest stand reconstruction will be addressed in future work.

vegetation phase: We are planning to repeat this study in different seasons. Especially in summer when trees are foliated results are likely to change dramatically. However, other ecological or forestry relevant parameters such as canopy closure or species detection might become measurable.

utilization of spectral information: One advantage of computer vision based point clouds over Lidar-Point clouds is that they are coming with spectral data. At this stage we didn't use this information, however we believe that this can improve results and becomes very valuable when using leaf-on data.

\section{ACKNOWLEDGEMENTS}

This study was supported by suedwest-geocopter by operating the octocopter and providing camera equipment.

\section{REFERENCES}

Asner, G. P., 2009. Tropical forest carbon assessment: integrating satellite and airborne mapping approaches. Environmental Research Letters 4(3), pp. 034009.

Bienert, A., Scheller, S., Keane, E., Mohan, F. and Nugent, C., 2007. Tree detection and diameter estimations by analysis of forest terrestrial laserscanner point clouds. In: Proceedings of ISPRS Workshop on Laser Scanning 2007 and SilviLaser 2007 , Espoo, September 12-14, 2007, Finland, Commission V, WG V/3, p. 5055. 
Eisenbeiss, H., 2009. UAV Photogrammetry. PhD thesis, ETH Zurich.

Frolking, S., Palace, M. W., Clark, D. B., Chambers, J. Q., Shugart, H. H. and Hurtt, G. C., 2009. Forest disturbance and recovery: A general review in the context of spaceborne remote sensing of impacts on aboveground biomass and canopy structure. Journal of Geophysical Research: Biogeosciences 114(G2), pp. n/a-n/a.

Furukawa, Y. and Ponce, J., 2010a. Accurate, dense, and robust multi-view stereopsis. IEEE Trans. on Pattern Analysis and Machine Intelligence 32(8), pp. 1362-1376.

Furukawa, Y. and Ponce, J., 2010b. Accurate, dense, and robust multiview stereopsis. Pattern Analysis and Machine Intelligence, IEEE Transactions on 32(8), pp. $1362-1376$.

Haala, N., Cramer, M., Weimer, F. and Trittler, M., 2011. Performance test on uav-based photogrammetric data collection. Proceedings of the International Archives of the Photogrammetry, Remote Sensing and Spatial Information Sciences XXXVIII1/C22, pp. 7-12.

Harwin, S. and Lucieer, A., 2012. Assessing the accuracy of georeferenced point clouds produced via multi-view stereopsis from unmanned aerial vehicle (uav) imagery. Remote Sensing 4(6), pp. 1573-1599.

Hongoh, D., Kajiwara, K. and Honda, Y., 2001. Developing ground truth measurement system using rc helicopter and brdf model in forest area. In: 22nd Asian Conference on Remote Sensing, Vol. 5, Sigapore, p. 9.

Hunt, E.Raymond, J., Cavigelli, M., Daughtry, C., Mcmurtrey, JamesE., I. and Walthall, C., 2005. Evaluation of digital photography from model aircraft for remote sensing of crop biomass and nitrogen status. Precision Agriculture 6(4), pp. 359-378.

Hyyppä, J., Hyyppä, H., Leckie, D., Gougeon, F., Yu, X. and Maltamo, M., 2008. Review of methods of small-footprint airborne laser scanning for extracting forest inventory data in boreal forests. International Journal of Remote Sensing 29(5), pp. 13391366.

Liang, X., Litkey, P., Hyyppa, J., Kukko, A., Kaartinen, H. and Holopainen, M., 2008. Plot-level trunk detection and reconstruction using one-scan-mode terrestrial laser scanning data. In: International Workshop on Earth Observation and Remote Sensing Applications, pp. $1-5$.

Lingua, A., Marenchino, D. and Nex, F., 2009. Performance analysis of the sift operator for automatic feature extraction and matching in photogrammetric applications. Sensors 9(5), pp. 3745-3766.

Lovell, J., Jupp, D., Newnham, G. and Culvenor, D., 2011. Measuring tree stem diameters using intensity profiles from groundbased scanning lidar from a fixed viewpoint. ISPRS Journal of Photogrammetry and Remote Sensing 66(1), pp. $46-55$.

Lowe, D., 2004. Distinctive image features from scale-invariant keypoints. International journal of computer vision 60(2), pp. 91110.

Lucieer, A., Robinson, S., Turner, D., Harwin, S. and Kelcey, J., 2012. Using a micro-uav for ultra-high resolution multi-sensor observations of antarctic moss beds. International Archives of the Photogrammetry, Remote Sensing and Spatial Information Sciences XXXIX-B1, pp. 429-433.
Merino, L., Caballero, F., Martnez-de Dios, J., Maza, I. and Ollero, A., 2012. An unmanned aircraft system for automatic forest fire monitoring and measurement. Journal of Intelligent \& Robotic Systems 65, pp. 533-548.

Moberg, L. and Nordmark, U., 2006. Predicting lumber volume and grade recovery for scots pine stems using tree models and sawmill conversion simulation. Forest products journal 56(4), pp. $68-74$.

Remondino, F., Barazzetti, L., Nex, F., Scaioni, M. and Sarazzi, D., 2011. Uav photogrammetry for mapping and 3d modelingcurrent status and future perspectives. International Archives of the Photogrammetry, Remote Sensing and Spatial Information Sciences 38, pp. 25-31.

Rosnell, T. and Honkavaara, E., 2012. Point cloud generation from aerial image data acquired by a quadrocopter type micro unmanned aerial vehicle and a digital still camera. Sensors 12(1), pp. 453-480.

Rusu, R. B., Marton, Z. C., Blodow, N., Dolha, M. and Beetz, M., 2008. Towards $3 \mathrm{~d}$ point cloud based object maps for household environments. robotics and autonomous systems. Robotics and Autonomous Systems 56, pp. 927-941.

Snavely, N., Seitz, S. M. and Szeliski, R., 2006. Photo tourism: Exploring photo collections in 3d. In: ACM Transactions on Graphics (Proceedings of SIGGRAPH 2006).

Tao, W., Lei, Y. and Mooney, P., 2011. Dense point cloud extraction from uav captured images in forest area. In: Spatial Data Mining and Geographical Knowledge Services (ICSDM), 2011 IEEE International Conference on, pp. 389 -392.

Tesfamichael, S., Ahmed, F., Van Aardt, J. and Blakeway, F., 2009. A semi-variogram approach for estimating stems per hectare in eucalyptus grandis plantations using discrete-return lidar height data. Forest Ecology and Management 258(7), pp. 1188-1199.

Turner, D., Lucieer, A. and Watsona, C., 2011. Development of an unmanned aerial vehicle (uav) for hyper resolution vineyard mapping based on visible, multispectral, and thermal imagery. In: Proceedings of the 34th International Symposium on Remote Sensing of Environment (ISRSE34),, Sydney, Australia.

Valente, J. a., Sanz, D., Del Cerro, J., Barrientos, A. and de Frutos, M., 2013. Near-optimal coverage trajectories for image mosaicing using a mini quad-rotor over irregular-shaped fields. Precision Agriculture 14, pp. 115-132.

Vierling, K. T., Vierling, L. A., Gould, W. A., Martinuzzi, S. and Clawges, R. M., 2008. Lidar: shedding new light on habitat characterization and modeling. Frontiers in Ecology and the Environment 6(2), pp. 90-98.

Wallace, L. O., Lucieer, A. and Watson, C. S., 2012. Assessing the feasibility of uav-based lidar for high resolution forest change detection. ISPRS - International Archives of the Photogrammetry, Remote Sensing and Spatial Information Sciences XXXIXB7, pp. 499-504.

Wu, C., Agarwal, S., Curless, B. and Seitz, S. M., 2011. Multicore bundle adjustment. In: Computer Vision and Pattern Recognition (CVPR), 2011 IEEE Conference on, IEEE, pp. 3057-3064.

Zhang, C. and Kovacs, J., 2012. The application of small unmanned aerial systems for precision agriculture: a review. Precision Agriculture 13, pp. 693-712. 